\section{Feature encoding and pattern classifications with sequentially presented Markov stimuli*}

\author{
BILL R. BROWN and CHARLES E. AYLWORTH \\ University of Louisville, Louisville, Kentucky 40208
}

The major objective of this experiment was to develop and evaluate a methodology designed to permit more direct assessment of the detailed processes involved in prototype abstraction. Thirty Ss participated in a task having the following characteristics: (1) classifications of Markov-generated stimuli sampled from two different populations, (2) controlled scanning of pattern features, (3) a measure of the degree to which pattern features were correctly identified, and (4) intermittent reproduction of pattern features abstracted from collections of mixed instances. Results showed that a significant number of the Ss learned to classify the stimuli into categories corresponding to generation rules and, at least partially, abstracted the population prototypes from these variable instances. The feature identification data suggested that the Ss who were unsuccessful in classifying the stimuli into the rule-defined categories used an inappropriate strategy for sampling pattern information upon which to base their classifications.

The term "pattern recognition" has come to denote the detection or selection of objects in the environment which merit classification and the subsequent assignment of these objects to representative categories (Evans, 1968). In this context, several investigators (Attneave, 1957; Evans, 1967a; Oldfield, 1954; Posner \& Keele, 1968) have theorized that successful pattern recognition is based on man's ability to encode representations of selected stimulus features or attributes that are statistically associated with the identification classes. These representations of stimulus features are commonly termed prototypes or schemata, and the encoding process is frequently referred to as "prototype abstraction" or "schema formation."

An extensive amount of research relevant to prototype encoding has been conducted using the Markov stimuli generated by the VARGUS 7 computer system (Evans, 1967b). The VARGUS 7 computer program produces histoform stimuli which are deviations from a prototype, where the prototype consists of a most-probable sequence of column heights, as determined by a seven-element Markov process. A population of instances, all of which are deviations from the same prototype, is termed a schema family; different schema families may be conceptualized as clusters of points in a multidimensional attribute space, and the centroid of each schema cluster constitutes the prototype for that population of instances. Constraint redundancy $\left(R_{c}\right)$, a measure discussed by Evans (1967c), measures the extent to which the

* This research was conducted by the Performance Research Laboratory and supported by a Department of Defense Project THEMIS contract, No.HC19-69-C-0009, under the Department of the Army. population of instances adheres to the prototype. The $\mathbf{R}_{\mathrm{c}}$ variable thus reflects the tightness or looseness of each schema cluster. Within a schema cluster, the degree to which a particular instance adheres to the prototype (i.e., the cluster centroid) is reflected by the proportion of schematic steps (POSS) measure developed by Bersted, Brown, and Evans (1968).

Several experiments (Brown \& Evans, 1969; Brown \& Dansereau, 1969; Brown \& Dansereau, 1970; Brown \& Rebbin, 1970; Brown, Walker, \& Evans, 1968) have demonstrated that $S s$ can learn to discriminate among VARGUS 7 stimuli that are distortions of different prototypes without the benefit of knowledge of results or external reinforcement. In the same context, Evans and Arnoult (1967) have shown that $S$ s are able to successfully classify $50 \%$ redundant instances into categories that are representative of the underlying prototypes and can do so without information as to the number of schema families represented in the task (Bersted, Brown, \& Evans, 1969). These results, coupled with Posner and Keele's (1968) findings that the presence of a prototype facilitates recognition memory, constitute a basis for inferring that prototype encoding, and the subsequent storage of individual instances in terms of their deviations from this basic pattern, represents an effective process for facilitating economy of memory and the use of retained information for recognizing complex patterns.

Although the studies mentioned above explicitly demonstrated that Ss can classify stimuli in a manner consistent with the underlying prototypes, their results provide little information about the detailed processes of feature encoding and identification involved in Ss' classification of instances into schema-defined categories. Consequently, one of the main objectives of the present experiment was to develop a pattern recognition task that would permit a more direct assessment of the relevance of the above processes to Ss' classifications of stimuli clustered in a multidimensional attribute space. The following requirements served as guidelines for the development of the task used here.

First, the task must assure that all of the features in any given stimulus have been attended to by the Ss prior to classification. None of the previous experiments dealing with schema formation have attempted to control the spatial-temporal input of individual stimulus features to the Ss. Failure of Ss to correctly classify or reproduce a stimulus in these tasks thus might be attributed to attentional factors, rather than to a failure to recognize critical pattern features.

Second, since the previously cited studies used only molar response measures, they provided little detailed information about the degree to which Ss detected relevant pattern features in making a classification response or about the rate at which these covarying stimulus characteristics were learned. Consequently, stimulus classifications should be accompanied by a measure reflecting the degree to which pattern features of each stimulus were correctly identified (i.e., rather than inferring the latter from the classification responses).

Third, a basic supposition of the prototype encoding model is that Ss extract information statistically associated with the schema classes represented in the task and that the pattern features extracted probably do not exemplify any particular stimulus [i.e., since the instances are all different variations of the prototype(s)]. Consequently, in order to obtain a direct measure of the degree to which distinct prototypes have been encoded from a collection of mixed instances sampled from different schema families, Ss must be required periodically to output at prescribed intervals the pattern features judged to be distinctly representative of the identification classes.

The purpose of the present experiment was to assess pattern-recognition performance in a task meeting the above requirements and in which there were VARGUS 7 instances sampled from two different schema families. Specifically, the study was intended to accomplish the following objectives: (1) to determine the degree to which Ss classify these probabilistically generated stimuli into categories consistent with the two schema rules, using only the information present in the instances themselves; (2) to determine the extent to which Ss correctly identify the pattern features present in each 
Table 1

Thirty VARGUS 7 Stimuli (Eight Elements Per Stimulus) Sampled from Schema 1 and Schema 2

\begin{tabular}{|c|c|c|c|c|c|c|c|c|c|c|c|c|c|c|c|}
\hline \multicolumn{8}{|c|}{ Schema 1} & \multicolumn{8}{|c|}{ Schema 2} \\
\hline 4 & 7 & 5 & 1 & 3 & 4 & 7 & 6 & 1 & 6 & 3 & 5 & 7 & 4 & 5 & 7 \\
\hline 4 & 7 & $\frac{5}{6}$ & 2 & 7 & 6 & 2 & 1 & 4 & 7 & 4 & 2 & 1 & 7 & $\frac{5}{4}$ & 2 \\
\hline 7 & 1 & 3 & 4 & $\underline{1}$ & 3 & 4 & $\frac{\overline{3}}{3}$ & 2 & $\overline{5}$ & 7 & 3 & 5 & $\overline{1}$ & 6 & 3 \\
\hline 2 & $\overline{5}$ & 1 & 7 & 6 & 2 & 5 & $\overline{1}$ & 6 & $\frac{5}{3}$ & $\frac{1}{5}$ & 3 & 5 & $\frac{5}{7}$ & 4 & 2 \\
\hline 6 & 2 & 5 & $\overline{1}$ & 3 & $\underline{3}$ & 4 & 4 & 4 & 2 & 4 & $\frac{\overline{2}}{2}$ & 1 & $\underline{3}$ & 5 & 7 \\
\hline 1 & 3 & 6 & 6 & 2 & $\overline{5}$ & 1 & $\overline{4}$ & 4 & 2 & $\overline{1}$ & 6 & 4 & $\overline{2}$ & 2 & 2 \\
\hline 4 & 7 & $\frac{\overline{2}}{2}$ & $\overline{5}$ & 1 & 3 & 4 & $\overline{1}$ & 3 & 5 & 4 & 2 & $\overline{1}$ & 6 & $\overline{3}$ & $\overline{5}$ \\
\hline 2 & 5 & $\overline{7}$ & 6 & 2 & 1 & 3 & 4 & 4 & 2 & $\frac{\overline{5}}{5}$ & 7 & 4 & 2 & 6 & 3 \\
\hline 6 & 2 & $\underline{\overline{3}}$ & 4 & 7 & $\frac{\overline{6}}{6}$ & 7 & 5 & 7 & 4 & $\frac{5}{6}$ & 3 & 5 & 7 & $\overline{5}$ & 2 \\
\hline 3 & 4 & $\overline{7}$ & 4 & 7 & 6 & $\overline{2}$ & $\overline{5}$ & 4 & 2 & $\overline{1}$ & 5 & 7 & 4 & $\overline{2}$ & $\overline{1}$ \\
\hline 2 & 5 & $\underline{2}$ & $\overline{5}$ & 1 & 4 & 7 & 6 & 7 & 4 & 2 & $\overline{1}$ & 6 & 6 & 3 & $\underline{3}$ \\
\hline 2 & 5 & $\overline{1}$ & 3 & 2 & $\overrightarrow{5}$ & $\underline{5}$ & 5 & 1 & 6 & 7 & 7 & 4 & $\overline{2}$ & 1 & $\underline{3}$ \\
\hline 1 & 3 & 4 & 7 & $\overline{6}$ & 2 & $\overline{\overline{7}}$ & $\overline{6}$ & 3 & 5 & $\overline{2}$ & $\dot{1}$ & 6 & 3 & 5 & $\frac{5}{7}$ \\
\hline 2 & $\underline{6}$ & 2 & 5 & 1 & $\underline{6}$ & $\overline{2}$ & 5 & 3 & 5 & $\overline{7}$ & 4 & $\underline{5}$ & 7 & 4 & 1 \\
\hline 5 & $\overline{7}$ & 6 & $\underline{4}$ & 7 & $\underline{I}$ & 3 & 4 & 5 & $\underline{1}$ & 6 & 3 & $\underline{\underline{1}}$ & 6 & 3 & $\underline{6}$ \\
\hline
\end{tabular}

stimulus prior to classification; and (3) to determine the degree to which pattern features, statistically associated with the schema categories, are abstracted from collections of instances over classification trials.

\section{Subjects}

\section{METHOD}

The Ss were 30 undergraduate students enrolled in introductory psychology courses at the University of Louisville.

\section{Stimuli}

The stimuli were 30 eight-element Markov sequences generated by the VARGUS 7 computer program at approximately $50 \% R_{c}$. This magnitude of $\mathbf{R}_{\mathrm{c}}$ was selected because all previous studies involving categorization of VARGUS 7 stimuli had utilized $50 \% \mathrm{R}_{\mathrm{c}}$. Two distinct families of stimuli were produced by creating variants of two prototypes which, as pointed out previously, were two most-probable sequences (MPSs) of digits varying from 1 to 7 . The MPS for Schema 1 was the sequence 3476251 , whereas the MPS for Schema 2 was 6357421 . Table 1 presents the 30 stimuli (15 per schema family). In each stimulus, the elements that constituted deviations from the basic MPS for each of the two schema families are placed in italics.

Each of the 30 VARGUS 7 stimuli was presented to $S s$ in the form of a mimeographed booklet. As shown in Fig. 1, each booklet consisted of eight separate pages $\left(5^{1 / 2} \times 4 \mathrm{in}\right.$.), and on each page there was printed a matrix of large dots having seven rows and eight columns. Each element of a given stimulus was mapped directly into one (and only one) matrix by circling the matrix dot representing the element. These elements are depicted in Fig. 1 by the large filled circles. The mapping rule used stated that the matrix row number represented the value of a particular stimulus element (ranging from one to seven), and the matrix column represented the position of the element in the stimulus. For example, as in the case of the stimulus depicted in Fig. 1, if the first element of the stimulus was the number " 4 ," then the dot in Row 4, Column 1 was circled on the first page of the booklet. Similarly, if the second element in the stimulus was " 7 ," then the dot in Row 7, Column 2 on the second page of the booklet was circled. This mapping process was continued through the eight booklet pages, with only one stimulus element being represented on each page. Thirty separate booklets were constructed using this mapping procedure, in order to represent the 30 distinct VARGUS 7 stimuli.

\section{Procedure}

Each of the Ss received the 30 stimulus booklets one at a time. The Ss were instructed to study carefully the circled element on the first page of the booklet for $5 \mathrm{sec}$ and to then predict the next element in the sequence by circling a dot in the subsequent column. In Fig. 1, a S's hypothetical responses are depicted by the unfilled circles. They were then instructed to turn the page and were given $5 \mathrm{sec}$ to check the correctness of their prediction, and this element then constituted the signal for their next prediction; the Ss made a response in the subsequent column, and so forth, until they had worked through the entire eight-element sequence. For each eight-element sequence, the Ss thus made seven predictions.

After checking the correctness of the last prediction in a booklet, the $S$ s were given $10 \mathrm{sec}$ to classify the sequence just seen as Type A or Type B. The Ss were instructed to label the first booklet Type A and were encouraged to be as consistent as possible in their use of category labels after

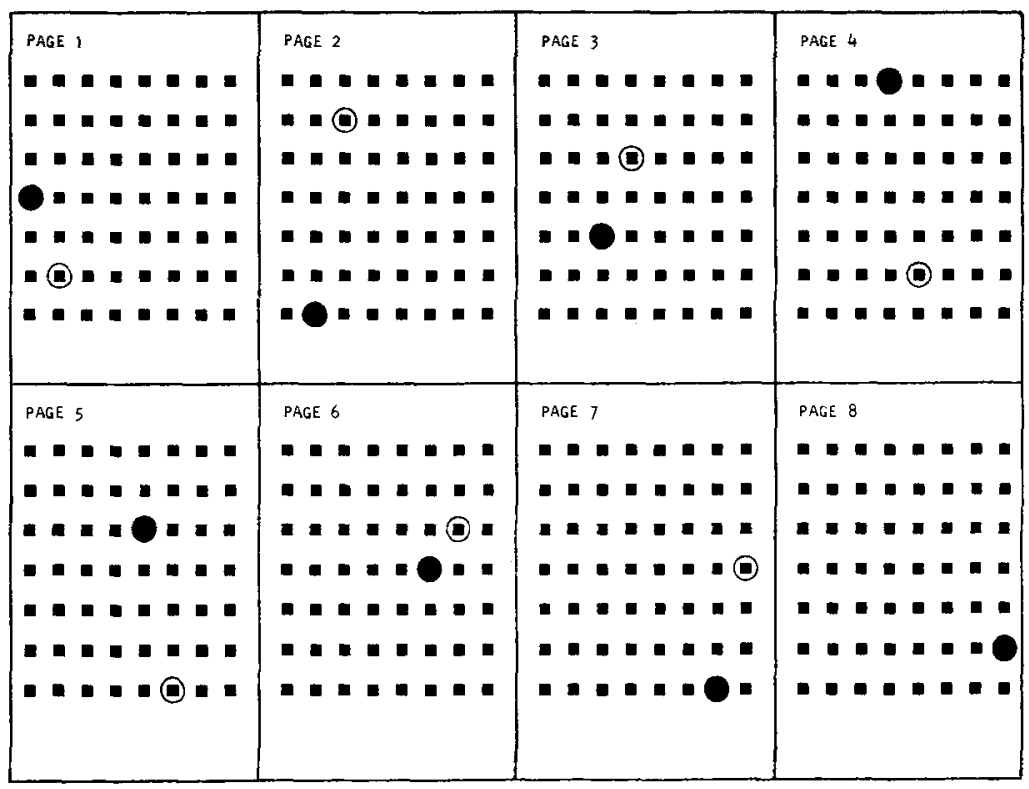

Fig. 1. Example of an eight-page VARGUS 7 stimulus booklet. The filled circles represent the stimulus 47513476 (a deviation of the MPS for Schema 1). The unfilled circles depict the responses (all erroneous in this case) of a hypothetical $S$ to the sequentially presented stimulus elements. 


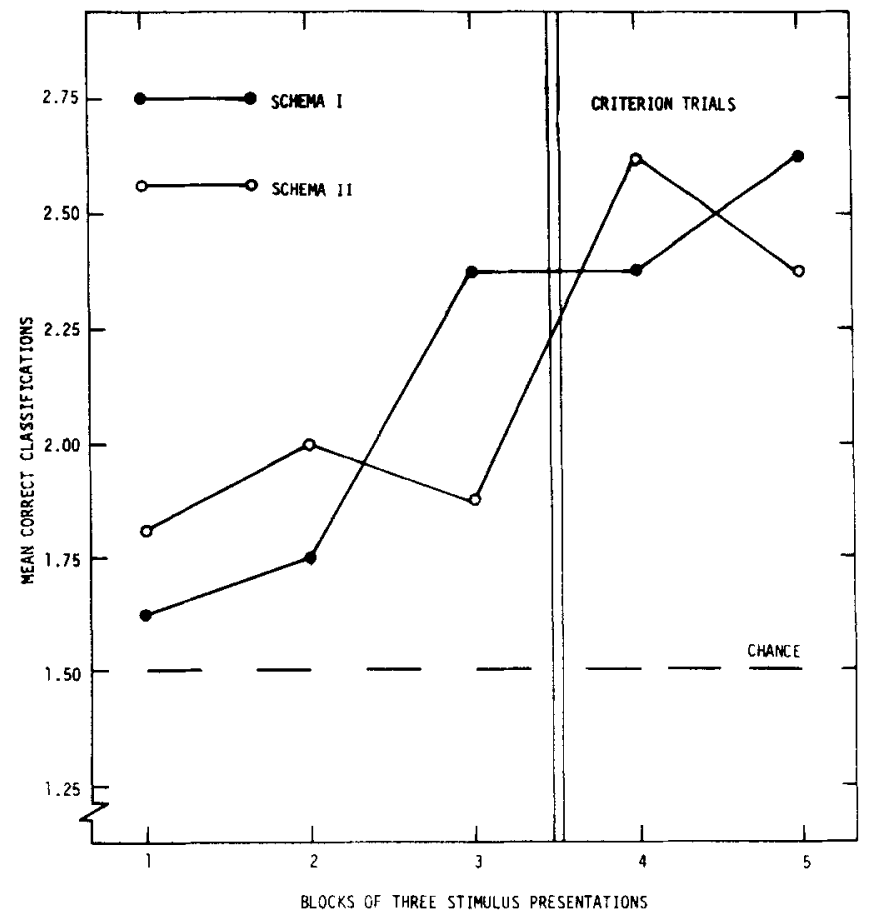

Fig. 2. Mean correct classifications (over five blocks of three trials) of stimuli sampled from Schema 1 and Schema 2 for the six Ss achieving the consistency criterion.

they had labeled the initial stimulus.

After working through and classifying every sixth stimulus booklet, the Ss were given a sheet having two blank 7 by 8 matrices. They were then instructed to reproduce (by circling one element in each of the eight matrix columns) what they thought the two basic sequences were for Pattern Category A and Pattern Category $\mathrm{B}$; the reproduced transitions could be initiated by the $S s$ at any point in the Markov sequences. Having attempted to reproduce the two sequences, the $\mathrm{Ss}$ labeled them $\mathbf{A}$ and $\mathbf{B}$, and were strongly encouraged to make these labels consistent with the labeling system used with respect to each of the preceding booklets.

The 30 stimulus booklets for each $\mathrm{S}$ were presented in a random order, except for the following constraints: (1) Each block of six booklets consisted of three stimuli sampled from Schema 1 and three from Schema 2 (although the order of presentation was randomized within a block); (2) the three values of POSS (i.e., $.96, .78, .65)$ were equally represented in the block of six booklets; and (3) POSS for any one block of six booklets was distributed equally with respect to the two schema families.

The $\mathrm{S}$ s participated in the experiment in groups of two and were told that they would be viewing different variations of two basic patterns (i.e., the term "schema family" was not used). No knowledge of results or external reinforcement was provided with respect to stimulus classifications or prototype reproduction, and $\mathrm{Ss}$ were encouraged to guess if necessary in making the various responses. The $\mathrm{Ss}$ were carefully prevented from looking at more than one booklet page at a time throughout the entire task.

\section{RESULTS \\ Stimulus Classification}

Since a single variable stimulus is not sufficient to define a schema family, the designation of the first instance as Type A would not necessarily prevent some Ss from reversing their labeling systems later in the task. Consequently, rather than scoring responses as correct or incorrect on the basis of the first instance being labeled Type A, classifications were scored on the basis of their consistency with either of the two labeling systems available to the Ss. A $S$ was considered to have classified the 30 stimuli in a manner consistent with the schema-defined categories if his responses matched either labeling system on at least 10 of the last 12 trials. Reference to the cumulative binomial distribution $(p=q=.50)$ shows that the probability of a $S$ satisfying this criterion while responding independently of the schema-defined categories is only .039 .

The results showed that 6 of the $30 \mathrm{Ss}$ achieved the criterion of consistency described above. The expected frequency as a result of Type 1 error would be approximately one, and reference to the binomial indicates the number of consistent $\mathrm{Ss}$ to be significantly larger than could be accounted for on the basis of Type 1 error. It thus may be concluded that at least $20 \%$ of the Ss classified the stimuli in a manner consistent with the two schema-defined categories represented in the task. This percentage is remarkably similar to those reported by several other experiments (Brown \& Evans, 1969; Evans \& Arnoult, 1967), which used a similar criterion of consistency.

Each of the consistent Ss' classifications throughout the entire task were scored as correct or incorrect in terms of the categorization rule he was apparently using during the last 12 trials. A two-way analysis of variance, with repeated measures on both factors (five blocks of three trials and the two schema-defined categories), was performed using total number of correct classifications as the dependent variable. Only the trials main effect $[F(4,20)=3.01, p<.05]$ was significant. Figure 2 shows that accuracy of classifications for Schema 2 was initially slightly superior to that for Schema 1 , but on the three trials immediately preceding the criterion trials, performance with instances from Schema 1 was higher.

In order to determine if the total number of correct classifications prior to the criterion trials was significantly different from chance, individual comparisons were made for each of the two schema families. Classification performance for Schema $1 \quad(t=2.61$, $\mathrm{df}=5, \mathrm{p}<.05$ ) was significantly better than chance, but performance for Schema 2 was not. It may thus be concluded that consistent classifications prior to criterion trials were specific to stimuli sampled from Schema 1. During the 12 criterion trials, however, exactly the same number of correct classifications occurred for each of the two schema families.

\section{Feature Identification}

In order to assess the degree to which the six consistent Ss successfully predicted the individual Markov transitions in the 30 stimuli, a two-way analysis of variance with repeated measures on the two factors (five blocks of three stimuli and the two schema families represented in the task) was computed using the number of correct predictions as the dependent variable. Only the blocks main effect $[F(4,20)=2.97$, $\mathrm{p}<.05]$ was significant in this analysis. Figure 3 shows that correct predictions for the consistent Ss increased as a function of trial blocks, and approximately the same level of accuracy was achieved for instances sampled from the two schema families. Moreover, the total number of correct classifications for both Schema 1 $(t=2.584, d f=5, p<.05)$ and Schema 2 $(t=2.62$, df $=5, p<.05)$ was significantly 


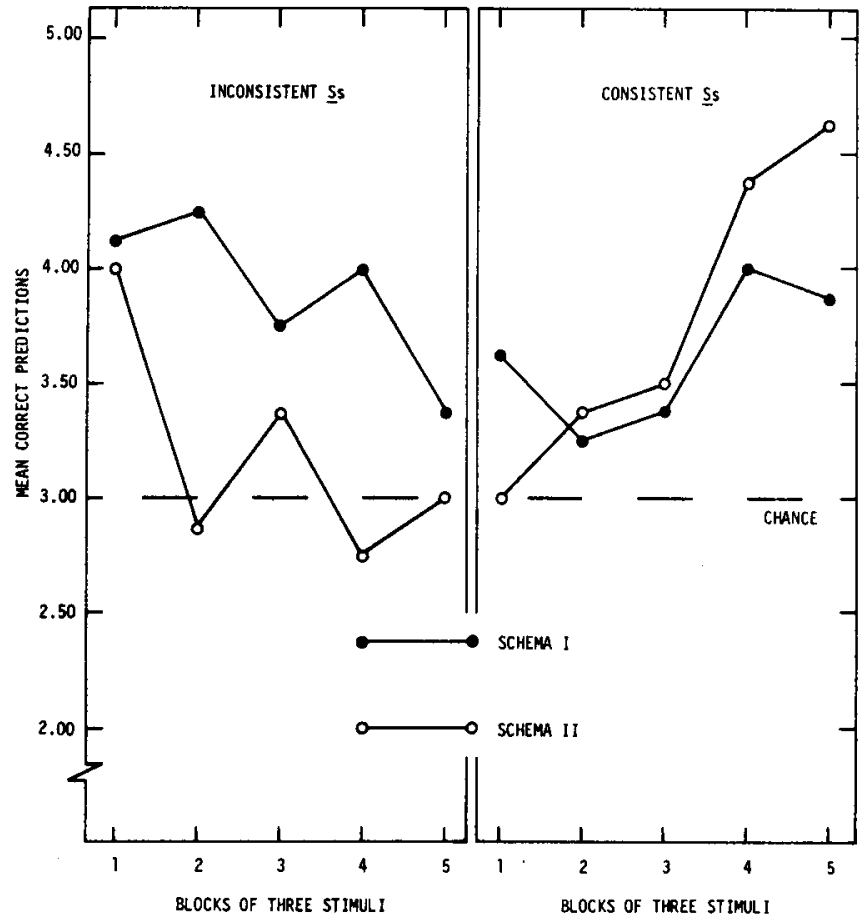

Fig. 3. Mean correct predictions of Markov elements (over five blocks of three stimulus presentations) exhibited by the 6 consistent Ss and the 24 inconsistent Ss for stimuli sampled from Schema 1 and Schema 2.

greater than chance.

Analyses identical to those described above were performed for the 24 Ss not meeting the consistency criterion. Both the main effect of the schema category factor $[F(1,23)=3.68, p<.05]$ and the Schema by Trial Blocks interaction $[F(4,92)=3.77, p<.05]$ were significant. Performance with instances sampled from Schema 1 . was significantly better than chance $(t=3.52, d f=23, p<.05)$, but the total number of correct predictions for Schema 2 did not differ significantly from chance level. Inspection of Fig. 3 shows that predictions for Schema 1 were more accurate than those for Schema 2, but that performance decreased as a function of trials for both schema-defined categories.

\section{Feature Reproduction}

Each $S$ on a reproduction trial was required to reproduce two basic sequences and to label them Type A or Type B; scoring involved point-by-point comparisons of the reproduced transitions to the MPSs representing the stimuli consistently labeled A and B in the trials prior to reproduction. Consequently, since the label assigned by a $S$ to a reproduced sequence defined which MPS was to be used in scoring, only the reproductions of the Ss meeting the consistency classification criterion could be evaluated (i.e., the labels used by the inconsistent Ss did not permit a distinction to be made between the reproduced sequences).

A two-way analysis of variance, with repeated measures on both factors (five reproduction trials and the two schema families), was performed, using the number of correct Markov transitions reproduced as the dependent variable. No significant results were obtained in this analysis. The total number of correct reproductions for Schema 1, however, was significantly greater than would be expected by chance $(\mathrm{t}=2.63, \quad \mathrm{df}=5, \mathrm{p}<.05), \quad$ but performance for Schema 2 was not. Figure 4 shows that at least initially there were far more correct reproductions for Schema 1 than for Schema 2, but in later trials a decrement occurred in the former.

\section{DISCUSSION}

The results reported here indicate that some Ss can learn to classify probabilistically generated stimuli in a manner consistent with the schema-defined categories and can do so without the benefit of external reinforcement, knowledge of results, or prior familiarization with the population prototypes. As pointed out previously, the percentage of Ss meeting the criterion for consistency is very similar to that observed in several other experiments (Brown \& Evans, 1969; Evans \& Arnoult, 1967) which used complex VARGUS 7 histoforms; this study thus has provided additional support for the supposition that Ss can select relevant pattern features and construct classification rules using only the information available in the clusters of covarying characteristics associated with the schema families.

The majority of the Ss did not achieve

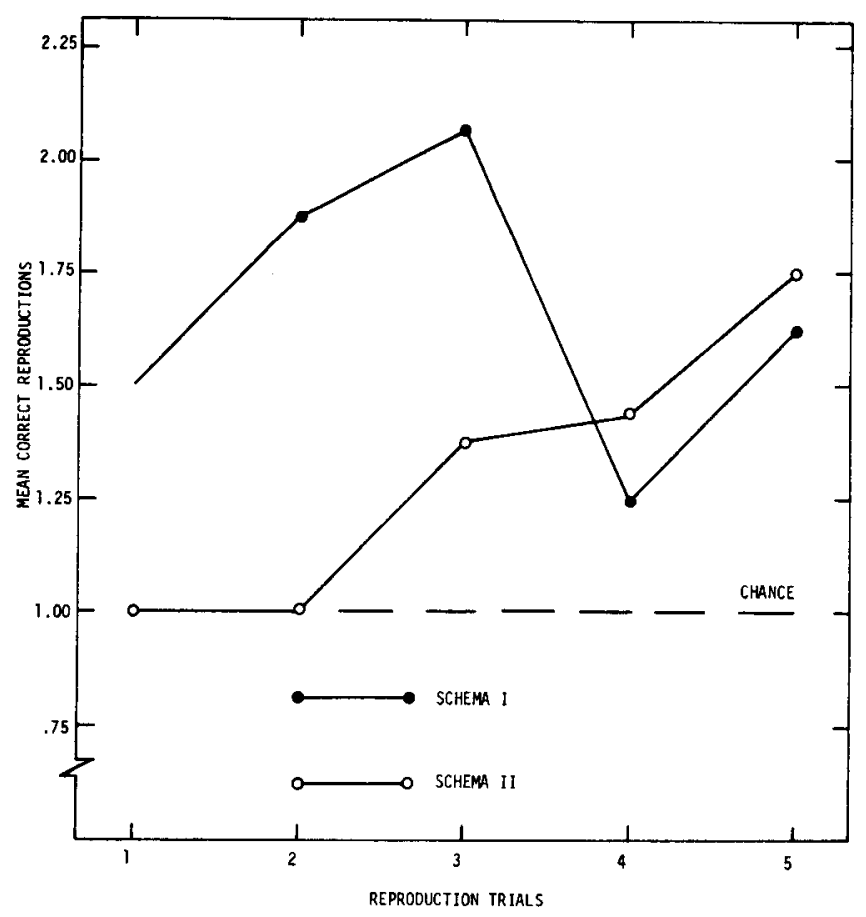

Fig. 4. Mean correct reproductions, scored against the most-probable sequences for Schema 1 and Schema 2, exhibited by the six consistent Ss over five reproduction trials. 
the criterion of consistency. There are several possible explanations of a methodological nature that should be considered with respect to this observation: (1) Since the criterion test was based on only 12 classification trials, it was not very powerful and, thus, could be expected to lead to a large number of false negatives (i.e., Type 2 error). (2) The magnitude of $R_{c}$ may not have been sufficiently high for some Ss to reliably assign stimuli to the appropriate categories. (3) The number of classification trials may not have been sufficiently large for feature encoding to occur to any great extent (4) The necessarily vague instructions as to what each $S$ was expected to do in the task may have been misinterpreted by many of the Ss. (5) The ambiguous nature of the task itself may have induced some Ss to adopt inappropriate classification rules as a means of eliminating the ambiguity.

Many of the above methodological considerations will likely prove relevant to the failure of Ss to consistently classify instances into schema-defined categories. The feature identification data, however, indicate what may be a more theoretically viable explanation for the fact that only some Ss met the consistency criterion. Correct predictions of individual stimulus elements exhibited by the consistent Ss generally increased over trials for both schema families, whereas exactly the opposite was observed for those Ss not meeting the consistency criterion. These results thus suggest that the two groups of Ss differed with respect to the manner in which they sampled the pattern information upon which their stimulus classifications were based. In this context, Evans (1964) has pointed out that the performance of Ss meeting the consistency criterion is characterized by low, gradual rates of information acquisition and that such gradual performance increments cannot be unequivocally interpreted as poor performance. Rather, it might be interpreted as a sampling process which ensures that the final results will be based on a large information sample by taking a small, but highly reliable, amount of information from each instance.

If stimulus features are imperfectly correlated with class membership (as in the case of the $50 \% \mathrm{R}_{\mathrm{c}}$ VARGUS 7 instances used here), a sampling strategy that involves taking large amounts of information from each variable stimulus would be expected to result in the acquisition of stimulus characteristics that have little statistical association with the schema families. The encoding of such irrelevant information would produce the increasingly decremental effect on the identification of stimulus features that characterized the performance of the inconsistent $S s$ and would be expected to prevent consistent classification of instances into the schema-defined categories.

The feature reproduction phase of the experiment indicated that the consistent Ss were able to successfully reproduce at least some aspects of the population prototypes represented in the task. There are, however, several points which require consideration in determining the theoretical implications of these findings. First, the reproduction data reported here is tenuous in that performance was exceedingly low for both Schema 1 and Schema 2. The low level of performance might be attributable to any of the methodological problems discussed earlier (e.g., a low magnitude of $R_{c}$ or the small number of trials), and it is clear that future studies using this methodology should utilize more optimal stimulus and task conditions.

Second, the consistent Ss, in effect, successfully reproduced only the stimulus features statistically associated with Schema 1. This observation is hardly surprising, however, in view of the fact that there were only two schema families represented in the task, and the encoding of one class prototype would be sufficient for successful classifications to occur (i.e., Ss could classify instances as $A$ or not $A$ or as B or not B). Therefore, further experiments should be conducted using this pattern-recognition task, in which there are three or more schema families represented.

Despite the limitations discussed above, it still may be concluded that the consistent Ss abstracted stimulus features, statistically associated with, and thus distinctively representative of, the schema classes, from collections of instances which were different variations of the population prototype(s). Since the Ss were never actually exposed to the undistorted prototypes, these results constitute explicit evidence for the prototype abstraction model discussed by Evans (1967c) and others, and provide the most direct support to date for the notion that the encoding of a prototype from variable stimuli and the subsequent storage of individual instances in terms of their deviations from this basic pattern represent processes which facilitate economy of memory and the use of retained information for recognizing complex patterns.

This experiment has indicated the pattern-recognition task developed here to be a potentially useful tool for the detailed assessment of the basic suppositions of the prototype-encoding model, especially in comparison to the more molar perceptual tasks used by the prior studies cited above. The incorporation of a larger number of classification trials and a higher magnitude of $R_{c}$ into the task, accompanied by the use of detailed verbal protocols and possibly more efficient feature reproduction procedures, should enhance the methodology's usefulness for determining the basic processes underlying prototype abstraction or schema formation and should contribute to the specification of the empirical determinants of the effectiveness of these processes for reducing environmental complexity.

\section{REFERENCES}

ATTNEAVE, $F$. Transfer of experience with a class-schema to identification learning of patterns and shapes. Journal of Experimental Psychology, 1957, 54, 81-88.

BERSTED, C. T., BROWN, B. R., \& EVANS, S. H. A standard set of VARGUS 7 patterns at three levels of schematic redundancy. Psychonomic Monograph Supplements, 1968, 2(13, Whole No. 29), 251-282.

BERSTED, C. T., BROWN, B. R., \& EVANS, S. H. Free sorting of stimuli clustered in a multidimensional attribute space. Perception \& Psychophysics, 1969, 6, 409-413.

BROWN, B. R., \& DANSEREAU, D. F. Discrimination among schematic stimuli as a function of response mode, constraint redundancy, and form of Markov rule. Psychonomic Science, 1969, 17, 197-198.

BROWN, B. R., \& DANSEREAU, D. F. Functional equivalence between same-different classifications and judged similarity of Markov patterns. Perception \& Psychophysics, 1970, 7, 307-310.

BROWN, B. R., \& EVANS, S. H. Perceptual learning in pattern discrimination tasks with two and three schema categories. Psychonomic Science, 1969, 15, 101-103.

BROWN, B. R., \& REBBIN, T. J. Simultaneous vs sequential discriminations of Markov-generated stimuli. Perception \& Psychophysics, 1970, 8, 355-359.

BROWN, B. R., WALKER, D. W., \& EVANS, S. H. Schematic concept formation as a function of constraint redundancy and knowledge of results. Psychonomic Science, 1968, 11, 75-76.

EVANS, S. H. A brief statement of schema theory. Psychonomic Science, 1967a, 8, 87-88.

EVANS, S. H. VARGUS 7: Computed patterns from Markov processes. Behavioral Science, 1967b, 12, 323-328.

EVANS, S. H. Redundancy as a variable in pattern perception. Psychological Bulletin, 1967c, 67, 104-113.

EVANS, S. H. An overview of machine and human pattern recognition. In Pattern identification by man and machine. Human Engineering Laboratories Technical Memorandum No. 17.68, Aberdeen Proving Ground, Maryland, 1968.

EVANS, S. H., \& ARNOULT, M. D. Schematic concept formation: Demonstration in a free sorting task. Psychonomic Science, 1967, 9, 221-222.

OLDFIELD, R. C. Memory mechanisms and the theory of schemata. British Journal of Psychology, 1954, 45, 14-23.

POSNER, M. I., \& KEELE, S. W. On the genesis of abstract ideas. Journal of Experimental Psychology, 1968, 77, 353-363.

(Accepted for publication July 6, 1970.) 